\title{
The Effect of Working Fluid Properties on the Performance of a Miniature Free Piston Expander for Waste Heat Harvesting
}

\author{
Sindhu Preetham Burugupally ${ }^{\mathrm{a}, *}$, Leland Weiss ${ }^{\mathrm{b}}$, Christopher Depcik $^{\mathrm{c}}$ \\ ${ }^{a}$ Department of Mechanical Engineering, Wichita State University, Wichita, KS 67260 USA \\ ${ }^{b}$ Department of Mechanical Engineering, Louisiana Tech University, Ruston, LA 71272 USA \\ ${ }^{c}$ Department of Mechanical Engineering, University of Kansas, Lawrence, KS 66045 USA
}

\begin{abstract}
Power generation from waste heat sources at the miniature length scales may be generated by using phase change working fluids (liquid-to-vapor) in a traditional boiler-expander-condenser-pump system. This paper builds on our prior work of boiler and expander design by investigating the effects of working fluid properties on an expander unit based on a Free Piston (FPE) architecture. Here, using first principles, a lumped-parameter model of the FPE is derived by idealizing the FPE as a linear spring-mass-damper system. Moreover, a linear-generator model is incorporated to study the effects on useful power output from the FPE directly. As a result, insight into the thermodynamic processes within the FPE are detailed and general recommendations for working fluid selection are established. They include: (1) to achieve a higher FPE efficiency, it is desirable for a working fluid to have high specific heat ratio, and (2) a peak output voltage of about $20 \mathrm{~V}$ AC and peak output power of around $2 \mathrm{~W}$ can be generated by coupling a centimeter-scale electromagnetic energy converter to the FPE. Overall, this effort shows the promise of reliable miniature thermal power generation from low temperature waste heat sources.
\end{abstract}

Keywords: Free piston, working fluids, waste heat harvesting, mathematical modeling, open cycle, linear generator.

\section{Introduction}

Miniature thermal power generation systems (centimeter size and below) have the flexibility to operate from a variety of available heat sources ranging from the low energy density waste heat from electronics to the high energy density of the heat of combustion generated from gaseous or liquid fuels 1, 2. This flexibility can be achieved using phase

\footnotetext{
* Corresponding author

Email addresses:

sindhupreetham. burugupally@wichita.edu (Sindhu

Preetham Burugupally), Iweiss@latech.edu (Leland Weiss), depcik@ku.edu (Christopher Depcik)
}

change working fluids (liquid-to-vapor) in a traditional boiler-expander-condenser-pump setup; here, the heat converts the phase of the working fluid - liquid into vapor - subsequently, increasing pressure that results in expander boundary work. For power generation at miniature length scales, lineartype expanders, such as reciprocating-type expanders (e.g. free pistons), may be a preferred choice as compared to rotary-type expanders (e.g. radial-flow turbines) due to their simple geometry, lower fabrication cost, and absence of high mechanical stresses induced by the rotary motion 3, 4. Moreover, the use of these reciprocating-type architectures addresses some of the existing technological challenges at the miniature length scale such as the issues arising from high- 
speed operation and sealing of rotational systems [5].

In macroscale reciprocating systems with a crankshaft assembly, the friction losses from the assembly can amount to more than $50 \%$ of the total friction loss [6]. Hence, upon scaling down the system size to a miniature length scale, friction losses can become dominant due to increased surface area to volume ratios and higher operating speeds [7. As a result, to reduce friction losses, free-piston based architectures are being developed [8, 9, 10] where the crankshaft assembly is completely eliminated. Subsequently, this removes crankshaft and bearing friction losses; thereby, improving the overall system efficiency. Furthermore, the low friction losses and pure linear motion of the piston nearly eliminates the need for lubrication. These advantages motivated researchers to investigate the performance of Free Piston Expanders (FPEs) coupled with linear alternators using mathematical models, simulations, and experiments 8. Overall, they show that it is possible to generate an output power $O\left(10^{1}\right) \mathrm{W}$ at relatively low operating frequencies $(<10 \mathrm{~Hz})$.

Like others 8, 11, the unique features of the freepiston architecture encouraged prior efforts at developing a FPE for waste heat recovery at the miniature length scales [3, 12. This previous work established many of the working principles [3, 12], as well as the technology demonstration of various mechanical components of the FPE [13, 14]. Generally, the centimeter-scale FPE under consideration (Fig. 11 has a unique potential to harvest waste heat. Specifically, the linear motion of the FPE allows coupling with a linear alternator for electricity generation similar to the work by Refs. [8, 15, 16]. Moreover, multiple boiler-expander units can be placed on waste heat sources where larger power output is necessary; whereas, single units can produce milli-Watt scale power for low power applications.

Typically, waste heat sources are of low "availability" or exergy such that the temperature gradient is relatively low [13. Therefore, it is desirable to employ working fluids with low boiling points (e.g., organic working fluids) [17. However, a proper choice of the working fluid is necessary for the optimal performance of the FPE. Different fluids (pure and mixtures), such as R-123 and R245fa, are being investi-
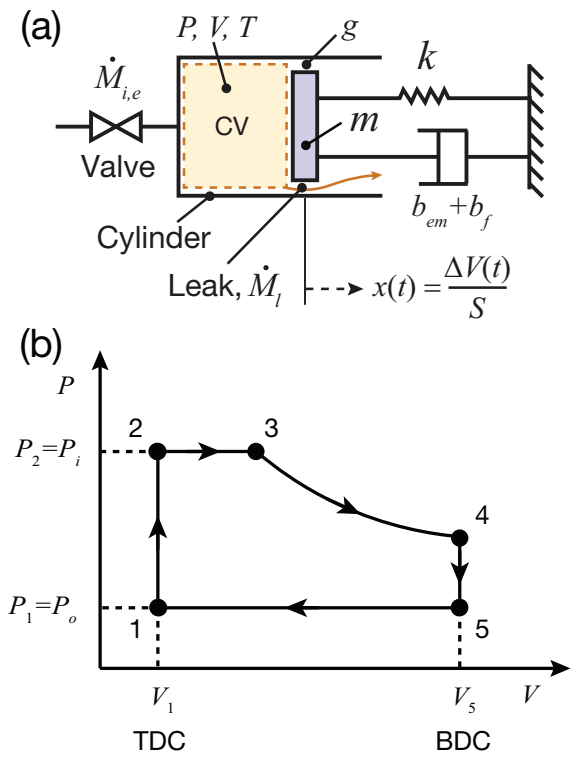

Figure 1: (a) A representation of FPE as a linear springmass-damper system. (b) Indicated pressure-volume diagram of the FPE.

gated as potential candidates [8, 15] due to a negative slope on the saturated vapor curve - hence called dry fluids - that enables superheating and expansion at a lower pressure while remaining 100\% vapor. This enables a greater output work from the FPE without the deleterious effects of condensed working fluids. As a result, these make an ideal choice for low temperature waste heat harvesting applications.

Therefore, this paper builds upon the prior foundation of boiler and FPE design through models and experiments [3, 13, 12, while concentrating on the effect of working fluid properties (Table 1) on the FPE performance. Unlike other approaches, where the nonlinear spring stiffness of the working fluid is linearized 18, the present study makes no such approximation. In this study, thermodynamic processes within the FPE are detailed and general recommendations for working fluid selection are established. Further, the effect of leakage losses within the FPE is studied, along with the determination of acceptable leakage losses. In addition, an electromagnetic energy conversion model is employed to simulate power output from the FPE. This provides an insight into 
useful power generation resulting from the working fluid study.

\section{Free Piston Expander}

\subsection{Design}

The FPE is a reciprocating-type expander similar to an internal combustion engine with a piston in-cylinder arrangement, however, devoid of the crankshaft assembly. Superheated vapor at a high pressure is injected into the cylinder control volume (CV) through a valve that is meant to displace the piston mass $(m)$ by a distance as a function of time $x(t)$ to produce boundary work (Fig. 17). Here, the $\mathrm{CV}$ denotes a control volume with state parameters: pressure $(P)$, volume $(V)$, and temperature $(T)$ at time $t$. The valve on the left is used for the injection $\left(\dot{M}_{i}\right)$ or exhaust $\left(\dot{M}_{e}\right)$ of the working fluid. Furthermore, this valve is connected to the high pressure working fluid line $\left(P_{i}, T_{i}\right)$ during the injection process and to the ambient atmosphere $\left(P_{o}, T_{o}\right)$ during the exhaust process. Moreover, the working fluid in the cylinder leaks out $\left(\dot{M}_{l}\right)$ through the piston-cylinder gap $(g)$. The spring of stiffness $k$ allows the horizontal displacement $x(t)$ of the piston. The working fluid trapped in the piston-cylinder gap introduces viscous friction modeled by a damper $\left(b_{f}\right)$ that impedes the motion of the piston. The mechanical energy of the piston will be converted into electrical energy via an electromagnetic energy converter modeled by a second damper $\left(b_{e m}\right)$. Generally, the absence of a crankshaft assembly mitigates friction losses and promotes less dependence on traditional lubricants. In fact, the working fluid may itself act as a lubricant 13. Unlike the standard reciprocating engines where Top Dead Center (TDC) and Bottom Dead Center (BDC) are geometrically set, the absence of crankshaft assembly allows the FPE to have a variable stroke length. Specifically, TDC and BDC for the FPE are determined by the operating conditions, that is load, time duration of injection process, and working fluid properties.

\subsection{Operation}

The FPE operates via an open cycle comprised of injection $(1 \rightarrow 2 \rightarrow 3)$, expansion $(3 \rightarrow 4)$, and ex- haust $(4 \rightarrow 5 \rightarrow 1)$ processes as depicted in Fig. 1 b. The cycle begins with the piston at state 1 (TDC) where superheated vapor at high pressure is injected into the cylinder $\mathrm{CV}$ by the opening of the valve. This results in pressurization of the CV and motion of the piston towards BDC. The valve remains open until the piston reaches state 3 . Note that the injection process $1 \rightarrow 2 \rightarrow 3$ occurs for a predetermined time duration $t_{13}$ obtained through parametric studies. Here, the process $1 \rightarrow 2$ occurs at constant volume, while the process $2 \rightarrow 3$ occurs at constant pressure $\sim P_{i}$. After the injection process, the valve closes and the expansion process $3 \rightarrow 4$ begins where the CV expands until state 4 . Next, the valve opens and the exhaust process starts with the expended working fluid discharged out of the $\mathrm{CV}$ to ambient pressure $P_{o}(4 \rightarrow 5 \rightarrow 1)$. This process comprises of the blowdown phase of exhaust $(4 \rightarrow 5)$, and exhaust displacement $(5 \rightarrow 1)$. During the displacement phase, the piston travels from BDC to TDC, subsequently, scavenging the remaining working fluid from the CV completing one operating cycle of the FPE.

\section{Model}

To investigate the effect of working fluid properties (Table 1) on FPE performance, a lumped-parameter model of the FPE is developed using first principles. Here, the FPE is modeled as a linear spring-massdamper system with a hollow cylinder CV containing the working fluid (vapor phase) (Fig. 119). When the displacement of the piston is zero, the cylinder volume $V_{o}$ is $V_{o}=L S$, where $L$ is the nominal cylinder length and $S$ is the cross-sectional area of the cylinder. Upon injecting the working fluid into the CV at temperature $T_{i}$ and pressure $P_{i}$, the piston displaces by $x(t)$. Then, a spring with stiffness $k$ helps restore this position $x(t)$ of the piston. Here, the movement of the piston is impeded by the dampers $b_{e m}$ and $b_{f}$, which model generated electrical energy and friction work, respectively. For steady state operation, the working fluid temperature, $T_{o}+\Delta T(t)$, pressure, $P_{o}+\Delta P(t)$, and cylinder volume, $V_{o}+\Delta V(t)$, undergo cyclic variation, where the nominal or ambient and time-varying cyclic components are denoted with the ' $O$ ' subscript and $\Delta$, respectively. 
Table 1: Nominal physical properties of different working fluids (vapor phase) in the operating range 298-323 K and 101-878 $\mathrm{kPa}$. The hypothetical fluid is used as an additional data point whose $\gamma$ equals that of the air and $c_{p}$ equals half that of the air [2009 ASHRAE Handbook Fundamentals (SI system)]

\begin{tabular}{|l|l|l|l|l|}
\hline Working fluid & $\begin{array}{l}\text { Specific } \\
\text { heat ratio, } \gamma\end{array}$ & $\begin{array}{l}\text { Specific heat at constant } \\
\text { pressure, } c_{p}(\mathrm{~J} / \mathrm{kg}-\mathrm{K})\end{array}$ & $\begin{array}{l}\text { Boiling } \\
\text { point }(\mathrm{K})\end{array}$ & $\begin{array}{l}\text { Dynamic viscosity, } \mu \\
(\mu \mathrm{Pa}-\mathrm{s})\end{array}$ \\
\hline Air ${ }^{1}$ & 1.40 & 1004 & 77 & 20 \\
R-123 (dry) & 1.15 & 786 & 300 & 404 \\
R-245fa (dry) & 1.25 & 1247 & 288 & 423 \\
R-134a (isentropic) & 1.32 & 1175 & 247 & 205 \\
Hypothetical & 1.40 & 502 & $<298$ & - \\
\hline
\end{tabular}

${ }^{1}$ Reference fluid

The lumped parameter model of the FPE shown in Fig. 17a is derived by applying Newton's second law for the piston along with the conservation of mass, conservation of energy, and the ideal gas model for the working fluid. Moreover, linear mass flow-rate relations for the valve and piston-cylinder gap, and linear electromagnetic damper are used [19], while also including a Newtonian fluid assumption for the lubricant (working fluid in liquid state). Mathematical statements of these principles are:

$$
\begin{gathered}
\frac{m}{S} \Delta \ddot{V}+\frac{b_{e m}+b_{f}}{S} \Delta \dot{V}+\frac{k}{S} \Delta V=S_{p} \Delta P \\
\dot{M}=\dot{M}_{i}-\dot{M}_{e}-\dot{M}_{l}
\end{gathered}
$$

$$
h \Delta T+\frac{\mathrm{d}\left(M c_{v} T\right)}{\mathrm{d} t}+P \Delta \dot{V}=\dot{M}_{i} c_{p} T_{i}-\left(\dot{M}_{e}+\dot{M}_{l}\right) c_{p} T,
$$

$$
P V=M R T
$$

$\dot{M}_{i, e}= \begin{cases}\dot{M}_{i}=\beta\left(P_{i}-P\right) ; \dot{M}_{e}=0, & \text { process } 1 \rightarrow 3 \\ \dot{M}_{i}=0 ; \dot{M}_{e}=0, & \text { process } 3 \rightarrow 4 \\ \dot{M}_{i}=0 ; \dot{M}_{e}=\beta\left(P-P_{o}\right), & \text { process } 4 \rightarrow 1\end{cases}$

$$
\begin{gathered}
\dot{M}_{l}=\chi \beta\left(P-P_{o}\right), \text { all processes } \\
b_{e m}=\frac{K_{v} K_{c}}{\Omega_{e x t}+\Omega_{i n t}}, \\
b_{f}=\frac{\mu S_{a}}{g},
\end{gathered}
$$

where $S_{p}$ is the piston cross-sectional area $(\approx 0.98 S)$, $M$ is the mass of the working fluid in the CV at any given time $t, h$ is a coefficient that models conduction/convection heat losses from the CV to the surroundings, $R$ is the mass-specific gas constant of the working fluid, $\beta$ is a proportionality constant, $\chi$ is a leakage loss factor $(\chi \in[0,1]), K_{v}$ and $K_{c}$ are the back electromotive force (EMF) constant and thrust constant, $\Omega_{\text {ext }}$ and $\Omega_{\text {int }}$ are external and internal resistive loads, $\mu$ is the dynamic viscosity of the working fluid, $S_{a}$ is the lateral surface area of the piston, $g(=0.01 \%$ cylinder bore $)$ is the gap between the piston and cylinder, and $c_{v}$ and $c_{p}$ are the constant volume and constant pressure heat capacities of the working fluid, respectively that are assumed constant over the temperature range. The overdot denotes a time derivative, while the subscripts ' $i$ ', ' $e$ ', and ' $l$ ' indicate injection, exhaust, and leakage processes, respectively. Like others [20], the load is assumed purely resistive in nature; that is, capacitance and inductance are zero.

If the working fluid injection pressure $P_{i}$, temper- 
ature $T_{i}$, time duration $t_{13}$, and initial conditions $\Delta V(0), \Delta P(0)$, and $\Delta T(0)$ are specified, Eqs. 1,8 constitute a nonlinear model for the determination of the intermediate thermodynamic state variables: $\Delta V(t), \Delta P(t)$, and $\Delta T(t)$. As the injection process is periodic, it is assumed that the dependent variables $\Delta V(t), \Delta P(t)$, and $\Delta T(t)$ will also be periodic under steady state conditions.

For solution, the nonlinear model is first nondimensionalized using the scales in Eq. 9

$$
\Delta \bar{V}=\frac{\Delta V}{V_{o}}, \Delta \bar{P}=\frac{\Delta P}{P_{o}}, \Delta \bar{T}=\frac{\Delta T}{T_{o}} \text {, and } \bar{t}=t \omega
$$

where $\omega=\sqrt{\frac{\gamma P_{o} S^{2}}{m V_{o}}}$ is a reference frequency, $\gamma$ is the specific heat ratio of the working fluid in vapor phase, and the overbar indicates a nondimensional independent or dependent variable. Thus, the obtained nondimensionalized equations are written in state space format as follows:

$$
\begin{gathered}
\frac{d \Delta \bar{V}}{d \bar{t}}=\Delta \dot{\bar{V}} \\
\frac{d \Delta \dot{\bar{V}}}{d \bar{t}}=\frac{S_{p} \Delta \bar{P}}{\gamma S}-\frac{b_{e m}+b_{f}}{m \omega} \Delta \dot{\bar{V}}-\frac{k}{m \omega^{2}} \Delta \bar{V} \\
\Delta \dot{\bar{T}}=\frac{1}{M c_{v} T_{o} \omega}\left[\dot{M}_{i} c_{p} T_{o}\left(1+\Delta \bar{T}_{i}\right)-\dot{M}_{i} c_{v} T_{o}(1+\Delta \bar{T})\right. \\
-\left(\dot{M}_{e}+\dot{M}_{l}\right) R T_{o}(1+\Delta \bar{T})-h T_{o} \Delta \bar{T} \\
\left.-P_{o} V_{o} \omega(1+\Delta \bar{P}) \Delta \dot{\bar{V}}\right]
\end{gathered}
$$$$
\Delta \dot{\bar{P}}=\frac{1}{P_{o} V_{o} \omega(1+\Delta \bar{V})}\left[-P_{o} V_{o} \omega(1+\Delta \bar{P}) \Delta \dot{\bar{V}}\right.
$$$$
\left.\left(\dot{M}_{i}-\dot{M}_{e}-\dot{M}_{l}\right) R T_{o}(1+\Delta \bar{T})+M R T_{o} \omega \Delta \dot{\bar{T}}\right]
$$

Here, it is noteworthy to mention that Eq. 13 is obtained by taking a first derivative of the ideal gas law Eq. 4 with respect to the nondimensional time $\bar{t}$.

To generate a pressure-volume diagram, the model Eqs. $10-13$ are numerically integrated in the order $1 \rightarrow 2 \rightarrow 3 \rightarrow 4 \rightarrow 5 \rightarrow 1$ (Fig. 1p) starting with state 1: $\Delta \bar{V}_{1}, \Delta \bar{P}_{1}, \Delta \bar{T}_{1}, \Delta \dot{\bar{V}}_{1}=0$. A proper choice of state variables at state $1: \Delta \bar{V}_{1}, \Delta \bar{P}_{1}, \Delta \bar{T}_{1}$ are necessary to obtain a steady state solution, and is obtained using a function-minimization algorithm presented in Refs. [9, 10]. The resulting volume $\Delta \bar{V}_{3}$, pressure $\Delta \bar{P}_{3}$, and temperature $\Delta \bar{T}_{3}$ from the injection process $(1 \rightarrow 2 \rightarrow 3)$ is computed by numerically integrating Eqs. 1013 from the initial condition $\Delta \bar{V}_{1}, \Delta \bar{P}_{1}, \Delta \bar{T}_{1}, \Delta \bar{V}_{1}=0$ for a predetermined time duration $\bar{t}_{13}$. The volumes, pressures, and temperatures $\Delta \bar{V}_{4}, \Delta \bar{P}_{4}, \Delta \bar{T}_{4}$ and $\Delta \bar{V}_{1}, \Delta \bar{P}_{1}, \Delta \bar{T}_{1}$ from the expansion $(3 \rightarrow 4)$ and exhaust $(4 \rightarrow 5 \rightarrow 1)$ processes are computed by numerically integrating Eqs. $10-13$ from the initial conditions $\Delta \bar{V}_{3}, \Delta \bar{P}_{3}, \Delta \bar{T}_{3}, \Delta \overline{\bar{V}}_{3}$ and $\Delta \bar{V}_{4}, \Delta \bar{P}_{4}, \Delta \bar{T}_{4}, \Delta \dot{\bar{V}}_{4}=0$ for times $\bar{t}_{34}$ and $\bar{t}_{41}$, respectively, such that the piston velocity $\Delta \dot{\bar{V}}_{4}=\Delta \dot{\bar{V}}_{1}=0$. It is worth noting that the times $\bar{t}_{34}$ and $\bar{t}_{41}$ are ' $a$ pri$o r i$ ' and are determined during the integration process. Note that injection time $\bar{t}_{13}$ (a nondimensional time) is chosen based on a parametric sweep on $\bar{t}_{13}$ ranging from $[0.001-10]$.

\section{Results and Discussion}

The centimeter-sized FPE shown in Fig. 17a modeled with $m=0.034 \mathrm{~kg}, k=1000 \mathrm{~N} / \mathrm{m}, K_{v}=30 \mathrm{~V}-\mathrm{s} / \mathrm{m}$, $K_{c}=33 \mathrm{~N} / \mathrm{A}, \Omega_{\text {ext }}=\Omega_{\text {int }}=50$ Ohms, $b_{f}=0 \mathrm{~N}-\mathrm{s} / \mathrm{m}$, $g=0.05 \mathrm{~mm}, h=0 \mathrm{~W} / \mathrm{K}, \beta=0.0064 \mathrm{~kg} / \mathrm{Pa}-\mathrm{s}, \chi=0.02$, $\bar{t}_{13}=3, S=0.785 \mathrm{~cm}^{2}, S_{p}=0.98 S \mathrm{~cm}^{2}, S_{a}=3.1 \mathrm{~cm}^{2}$ (0.99 $\mathrm{cm}$ in diameter and $1 \mathrm{~cm}$ of nominal length), and $V_{o}=0.785 \mathrm{~cm}^{3}(1 \mathrm{~cm}$ in diameter and $1 \mathrm{~cm}$ of nominal length) is treated as the reference case. The numerical values of some of the FPE parameters are based on prior work by the authors [10, 13, 12] and the electromagnetic damper parameters are chosen based on Ref.[19]. Standard temperature and pressure conditions of $T_{o}=298 \mathrm{~K}$ and $P_{o}=101 \mathrm{kPa}$ are chosen for the ambient state. The reference working fluid is compressed air, assumed to behave like 

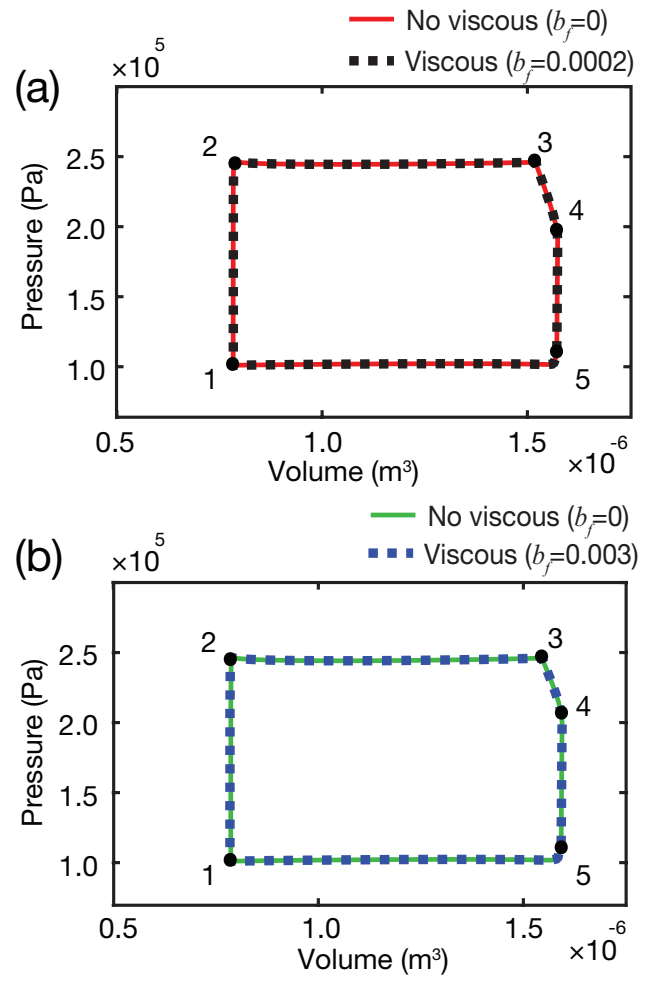

Figure 2: Effect of fluid viscosity on FPE operation for the reference case for (a) air (b) R-245fa working fluids with operating conditions: $P_{i}=250 \mathrm{kPa}, T_{i}=323 \mathrm{~K}, \bar{t}_{13}=3$, and $\chi=0.02$. The indicated thermal efficiencies for air and R-245fa are $11.73 \%$ and $7.99 \%$, while the corresponding Carnot efficiencies are $27.64 \%$ and $18.54 \%$, respectively. Note the unit of frictional damping coefficient $b_{f}$ is $\mathrm{N}-\mathrm{s} / \mathrm{m}$.

an ideal gas with $\gamma=1.4$ and $c_{p}=1004 \mathrm{~J} / \mathrm{kg}-\mathrm{K}$. It is noteworthy to mention that for the reference case, the electromagnetic damping coefficient is $b_{e m}=10 \mathrm{~N}$ s/m (Eq. 7).

\subsection{Pressure-volume diagram}

A P-V diagram of the FPE for one operating cycle $1 \rightarrow 2 \rightarrow 3 \rightarrow 4 \rightarrow 5 \rightarrow 1$ for the reference case with $T_{i}=$ $323 \mathrm{~K}, P_{i}=250 \mathrm{kPa}, \bar{t}_{13}=3$, and air as the working fluid is shown in Fig. 2 a (no viscosity, $b_{f}=0 \mathrm{~N}$ $\mathrm{s} / \mathrm{m})$. The first phase of the injection process $(1 \rightarrow 2)$ occurs at constant volume $\left(V_{1 \rightarrow 2}=0.785 \mathrm{~cm}^{3}\right)$ unlike the second phase $(2 \rightarrow 3)$ happens at constant pressure $(245 \mathrm{kPa})$ that results in the volume increase of the
$\mathrm{CV}, V_{3}=1.53 \mathrm{~cm}^{3}$. Following the injection process $(1 \rightarrow 2 \rightarrow 3)$, the FPE expands isentropically $(3 \rightarrow 4)$ to about $V_{4}=1.57 \mathrm{~cm}^{3}$, where the pressure is above atmosphere $\left(P_{4}=190 \mathrm{kPa}\right)$. Next, the blowdown phase $(4 \rightarrow 5)$ of the exhaust process $(4 \rightarrow 5 \rightarrow 1)$ occurs, where the expended working fluid is discharged due to the pressure gradient across the CV and exhaust system - a process that is nearly constant volume (1.57 $\left.\pm 0.01 \mathrm{~cm}^{3}\right)$. Finally, the FPE undergoes the displacement phase of the exhaust process $(5 \rightarrow 1)$, where the residual working fluid is scavenged from the $\mathrm{CV}$ until its volume becomes $V_{1}=0.781 \mathrm{~cm}$-a process that is nearly constant pressure $\left(P_{o} \pm 1.2 \mathrm{kPa}\right)$.

The constant pressure and constant volume processes are dictated by the constriction in the valve that controls working fluid flow rate to the expander (quantified by $\beta$ ). In this case, the larger the restriction (low $\beta$ ) in the valve, the greater the deviation from constant pressure or constant volume conditions (see Appendix Fig. A1). This enables the ability to incorporate tuned inlet and outlet passages through 3-D printing capabilities beneficial in The prevalence of 3-D printing makes it a natural choice to allow quick fabrication and validation of different potential port designs. This is especially true given the low temperature operating ranges of the investigated FPE and wide selection of plastic materials for consideration.

Overall, the ratio of the numerical integration of the P-V diagram (boundary work) to the enthalpy added $\left(M_{3} c_{p} T_{i}-M_{1} c_{p} T_{i}\right)$ over one operating cycle gives an efficiency of $11.72 \%$ (Fig. 22a).

\subsection{Effect of working fluid viscosity}

As stated prior, the damper $b_{f}$ models the viscous friction of the working fluid trapped between the piston-cylinder gap (Fig. 112). Here, two viscous fluids, namely air (low viscosity) and R-245fa (high viscosity), are studied to investigate the effect of fluid viscosity on FPE performance. For this, P$\mathrm{V}$ diagrams of the FPE for the reference case with $T_{i}=323 \mathrm{~K}, P_{i}=250 \mathrm{kPa}$, and $\bar{t}_{13}=3$ are plotted (Fig. 22). The P-V diagrams show that there is no significant difference between a FPE with $\left(b_{f}=0.0003\right.$ $\left.\mathrm{N}-\mathrm{s} / \mathrm{m} ; b_{f}=0.002 \mathrm{~N}-\mathrm{s} / \mathrm{m}\right)$ and without fluid friction $\left(b_{f}=0 \mathrm{~N}-\mathrm{s} / \mathrm{m}\right)$. Regardless of the fluid, it has been 


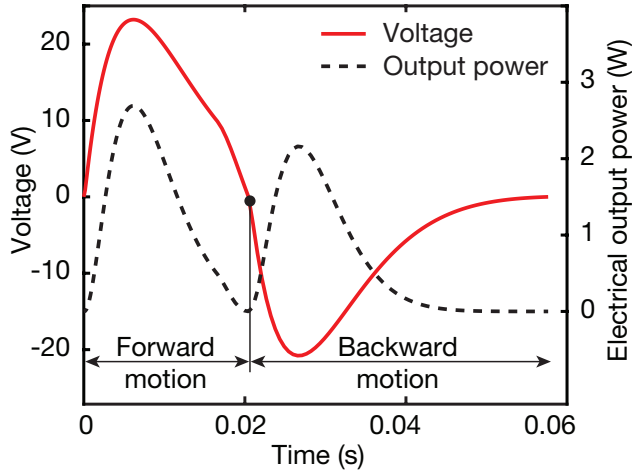

Figure 3: Time trace of generated voltage and electrical output power from the FPE for the reference case with operating conditions: $P_{i}=250 \mathrm{kPa}, T_{i}=323 \mathrm{~K}, \bar{t}_{13}=3$, and $\chi=0.02$. Note that load on the FPE is purely resistive with $\Omega_{e}=\Omega_{i}=50$ Ohms.

found that the difference in efficiency between the two cases: with $\left(b_{f} \neq 0\right)$ and without viscous friction $\left(b_{f}=0\right)$ is negligible $(<0.15 \%)$. This is due to the fact that the load $b_{e m}$ on the FPE is at least $O\left(10^{3}\right)$ higher than the fluid friction $b_{f}$. Therefore, the subsequent analyses, only consider $b_{e m}$ as the load on FPE (that is $b_{f}=0 \mathrm{~N}-\mathrm{s} / \mathrm{m}$ ).

\subsection{Generated voltage and electrical output power}

The instantaneous voltage generated by the electromagnetic energy converter connected to the FPE is equal to the product of the back electromotive force (EMF) constant $\left(K_{v}\right)$ and the instantaneous velocity of the piston. Therefore, the voltage is positive for the forward motion and negative for the backward motion of the piston, respectively (Fig. 3). For the reference case, a peak output $\mathrm{AC}$ voltage of $22 \mathrm{~V}$ and peak output power of $2.7 \mathrm{~W}$ can be generated. This offers a promise of reliable power generation at the miniature length scale.

\subsection{Effect of operating conditions}

The operating conditions, namely working fluid injection temperature $T_{i}$ and time duration $\bar{t}_{13}$, on the performance of the FPE is characterized in terms of efficiency (Figs. 4, 5). For this, the injection duration $\bar{t}_{13}$ is varied while holding $T_{i}$ constant and vice versa. Two observations can be made: (1) for a fixed
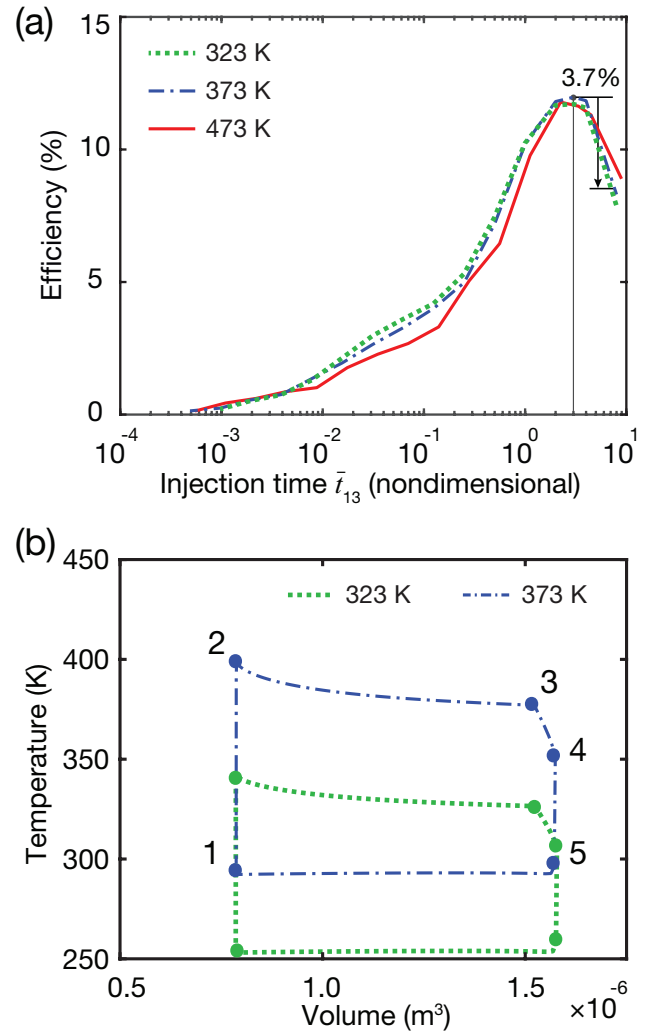

Figure 4: (a) Effect of operating conditions: $T_{i} \in$ $\{323,373,473\} \mathrm{K}$ and $\bar{t}_{13}$ on FPE efficiency for the reference case with $P_{i}=250 \mathrm{kPa}, \chi=0.02$, and air as the working fluid. Regardless of the working fluid injection temperature $T_{i}$, the FPE efficiency is nearly constant and peaks at $\bar{t}_{13}=3$ and then drops to about $3.7 \%$ for $\bar{t}_{13}=8$. (b) Temperature-Volume diagram for the reference case with $T_{i} \in\{323,373\} \mathrm{K}, \bar{t}_{13}=3$, $P_{i}=250 \mathrm{kPa}$, and $\chi=0.02$. The indicated thermal efficiencies for $T_{i}=323 \mathrm{~K}$ and $T_{i}=373 \mathrm{~K}$ are $11.73 \%$ and $11.97 \%$, while the corresponding Carnot efficiencies are $27.64 \%$ and $28.15 \%$, respectively.

$\bar{t}_{13}$, an increase in $T_{i}$ does not significantly affect the efficiency (Fig. 4). For instance, a FPE with injection temperatures $T_{i}=323 \mathrm{~K}$ and $373 \mathrm{~K}$ had same enthalpy addition of approximately $0.95 \mathrm{~J} /$ cycle, which resulted in same indicated thermal efficiency of approximately $12 \%$; and (2) regardless of the working fluid, an increase in $\bar{t}_{13}$ results in an initial increase in the FPE efficiency, which peaks at $\bar{t}_{13}=3$ and then drops (Fig. 5). This indicates that there is an opti- 


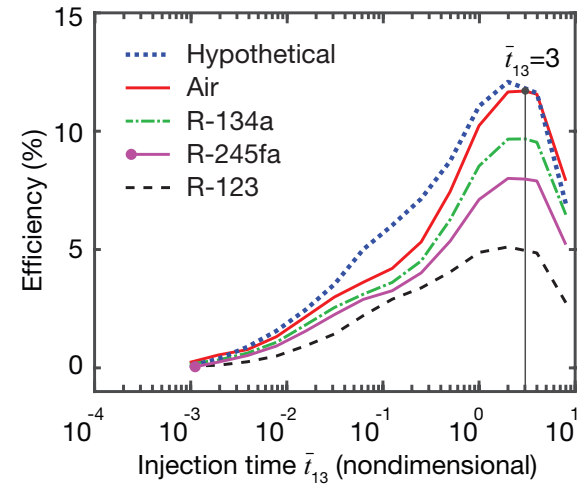

Figure 5: Effect of injection time $\bar{t}_{13}$ on the FPE efficiency for the reference case with different working fluids, $P_{i}=250 \mathrm{kPa}$, $T_{i}=323 \mathrm{~K}$, and $\chi=0.02$. Regardless of the working fluid, the FPE efficiency peaks at $\bar{t}_{13}=3$.

mal injection time duration, which maximizes energy conversion efficiency of the FPE. Therefore to maximize the efficiency, for the subsequent analyses, we chose $\bar{t}_{13}=3$.

\subsection{Effect of leakage loss}

The leakage loss from the FPE modeled by the factor $\chi$ adversely affects the efficiency (Figs. 6, 7). Specifically, an increase in $\chi$ reduces the amount of working fluid in the cylinder and the cylinder pressure, causing shorter displacement strokes $\left(V_{2} \rightarrow V_{4}\right)$. Hence, this decreases the amount of boundary work, while adversely lowering efficiency (Fig. 6). At the same time, the presence of leakage loss reduces the expansion stroke $\left(V_{3} \rightarrow V_{4}\right)$, consequently decreasing the expansion time $t_{34}$, while increasing the operating frequency $f$ (Fig. 6e inset). A TemperatureEntropy diagram of the FPE for one operating cycle $1 \rightarrow 2 \rightarrow 3 \rightarrow 4 \rightarrow 5 \rightarrow 1$ for the reference case with $T_{i}=323 \mathrm{~K}, P_{i}=250 \mathrm{kPa}, \bar{t}_{13}=3$, and air as the working fluid for different leakage losses $(\chi)$ is shown in Fig. 6b. The first phase of the injection process $(1 \rightarrow 2)$ adds entropy into the control volume; whereas, the second phase $(2 \rightarrow 3)$ lowers the entropy since the piston is expanding the working fluid in parallel. The expansion process $(3 \rightarrow 4)$ is isentropic, where the entropy of the CV remains constant. Following the expansion process, the blowdown phase
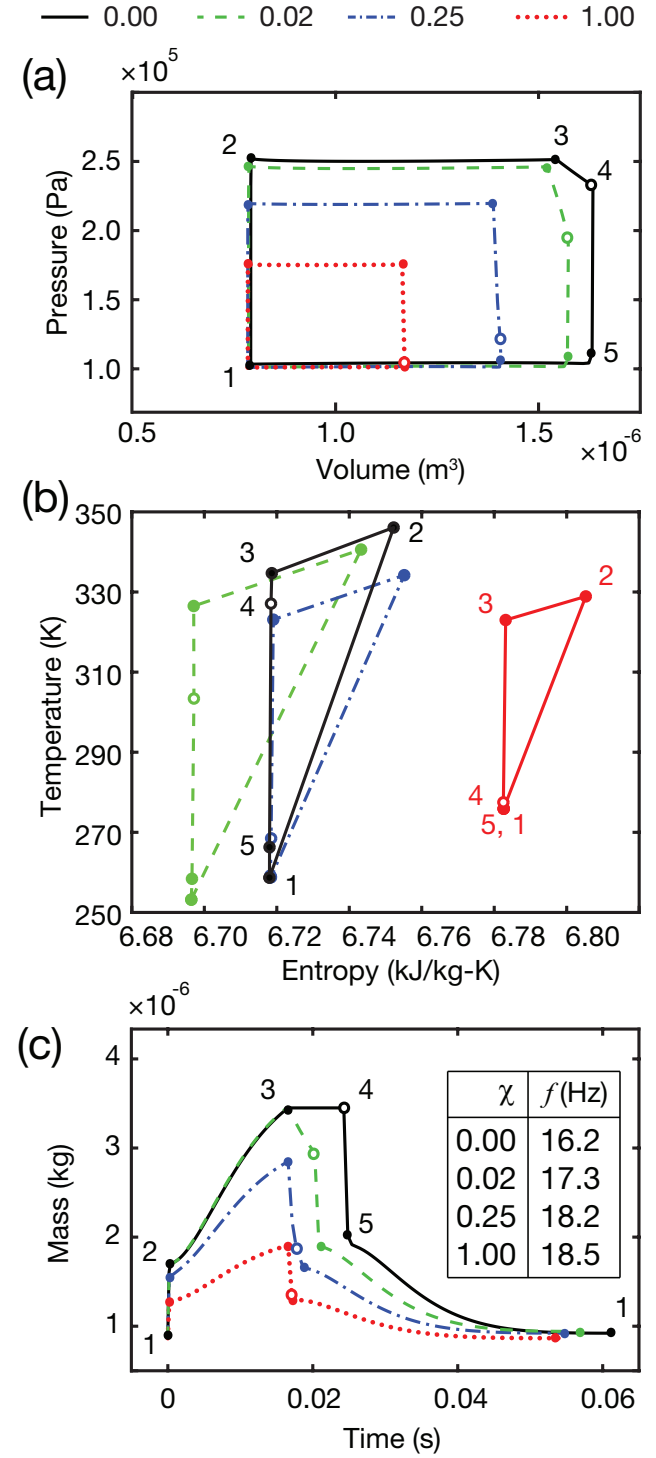

Figure 6: FPE behavior for the reference case with different leakage loss factors $\chi \in\{0,0.02,0.25,1\}, P_{i}=250 \mathrm{kPa}, T_{i}=323$ $\mathrm{K}, \bar{t}_{13}=3$, and air as the working fluid. (a) Pressure-Volume (b) Temperature-Entropy (c) Working fluid mass-Time plots for one operating cycle. The open circles 'o' correspond to state 4 . The operating frequency $f$ of the FPE for different $\chi$ is shown in the inset. Note that higher the $\chi$, higher will be the leakage loss from the FPE which results in smaller output work. A value $\chi=1$ implies that the amount of fluid leakage equals fluid lost from the exhaust valve. 


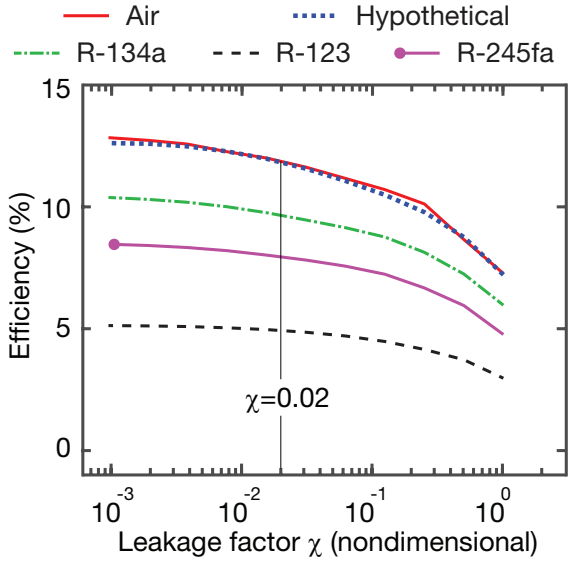

Figure 7: Effect of leakage loss $\chi$ on the FPE for the reference case operating with $P_{i}=250 \mathrm{kPa}, T_{i}=323 \mathrm{~K}$, and $\bar{t}_{13}=3$ for different working fluids.

$(4 \rightarrow 5)$ of the exhaust process occurs relatively quick and therefore this process is nearly isentropic (note: for conventional internal combustion engines Ref. [6] assumes the blowdown process as isentropic). Finally, the FPE undergoes the displacement phase of the exhaust process $(5 \rightarrow 1)$, where cycle reversion occurs. For the chosen working fluids, the drop in efficiency for $\chi=10^{-3}$ to $\chi=10^{0}$ is about $5.6 \%$ as illustrated in Fig. 7. To understand further, a P-V diagram for $\chi=0.02$ for the different working fluids is shown in Fig. 8. Though the area enclosed by the P-V diagrams are nearly same $(0.11 \mathrm{~J})$, the enthalpy added to the FPE is different for dissimilar fluids. In addition, Fig. 8 presents the first law, Carnot, and second law efficiencies highlighting that both the first law and Carnot efficiencies are respectively low due to a relatively cold high temperature reservoir. Computing the reversible work and finding that the cycle is able to achieve around $40 \%$ of this theoretical output (that is, second law efficiency) demonstrates the potential of this design. However, the assumption of adiabatic processes was made for this cycle; hence, future work will investigate the correct heat transfer expressions to utilize that will subsequently diminish both first and second law efficiencies. Note that the exhaust temperature from the FPE is $273 \mathrm{~K}$ and $292 \mathrm{~K}$ for R-245fa and R-123, respectively. These temperatures are $15 \mathrm{~K}$ and $8 \mathrm{~K}$ below their boiling points measured at standard atmospheric pressure. But, since R-245fa and R-123 are dry fluids, they are less likely to condense during the exhaust process $4 \rightarrow 5 \rightarrow 1$.

\subsection{Choice of working fluid}

A proper choice of working fluid characterized in terms of $\gamma$ and $c_{p}$ is essential for the optimal performance of the FPE. Different $\gamma \in[1.04,1.40]$ and $c_{p} \in[500,1400] \mathrm{J} / \mathrm{kg}-\mathrm{K}$ values are used in the model Eqs. 1013 to generate the P-V diagrams and calculate the corresponding efficiencies for $T_{i}=323 \mathrm{~K}$, $P_{i}=250 \mathrm{kPa}, \bar{t}_{13}=3$, and $\chi=0.02$ (Fig. 9). Two observations are made here. One, an increase in $\gamma$, increases the efficiency of the FPE proportionally. Two, $c_{p}$ has no significant effect on the efficiency. Therefore, to achieve a higher FPE efficiency, it is desirable for a working fluid in vapor phase to have high a $\gamma$. While a working fluid such as air is attractive based on these simulations, it is important to recognize that air will not have the same thermal absorption benefits of other potential working fluids. This is a critical consideration for the FPE system as a whole. In the complete system, thermal energy is scavenged from the surroundings via boiler or heat exchanger and transferred to the working fluid. A low boiling point refrigerant such as $3 \mathrm{M}^{\mathrm{TM}}$ PF5060DL (boiling point of $329 \mathrm{~K}$ ) or R-123 (boiling point of $300 \mathrm{~K}$ ) can undergo phase change from liquid to vapor in low temperature scavenging applications. This allows more energy absorption to the working fluid from the surroundings. Hence, careful consideration of the system as a whole is important in final working fluid selections.

\section{Conclusions}

Power generation from the waste heat sources at the miniature length scales can be generated by using phase change working fluids (liquid-to-vapor) in a boiler-expander-condenser-pump system. This paper focuses on the effect of working fluid properties on the expander unit, a Free Piston Expander (FPE). For this, a lumped-parameter model of the FPE is derived using first principles, where the FPE is modeled as a linear spring-mass-damper system. Three important 

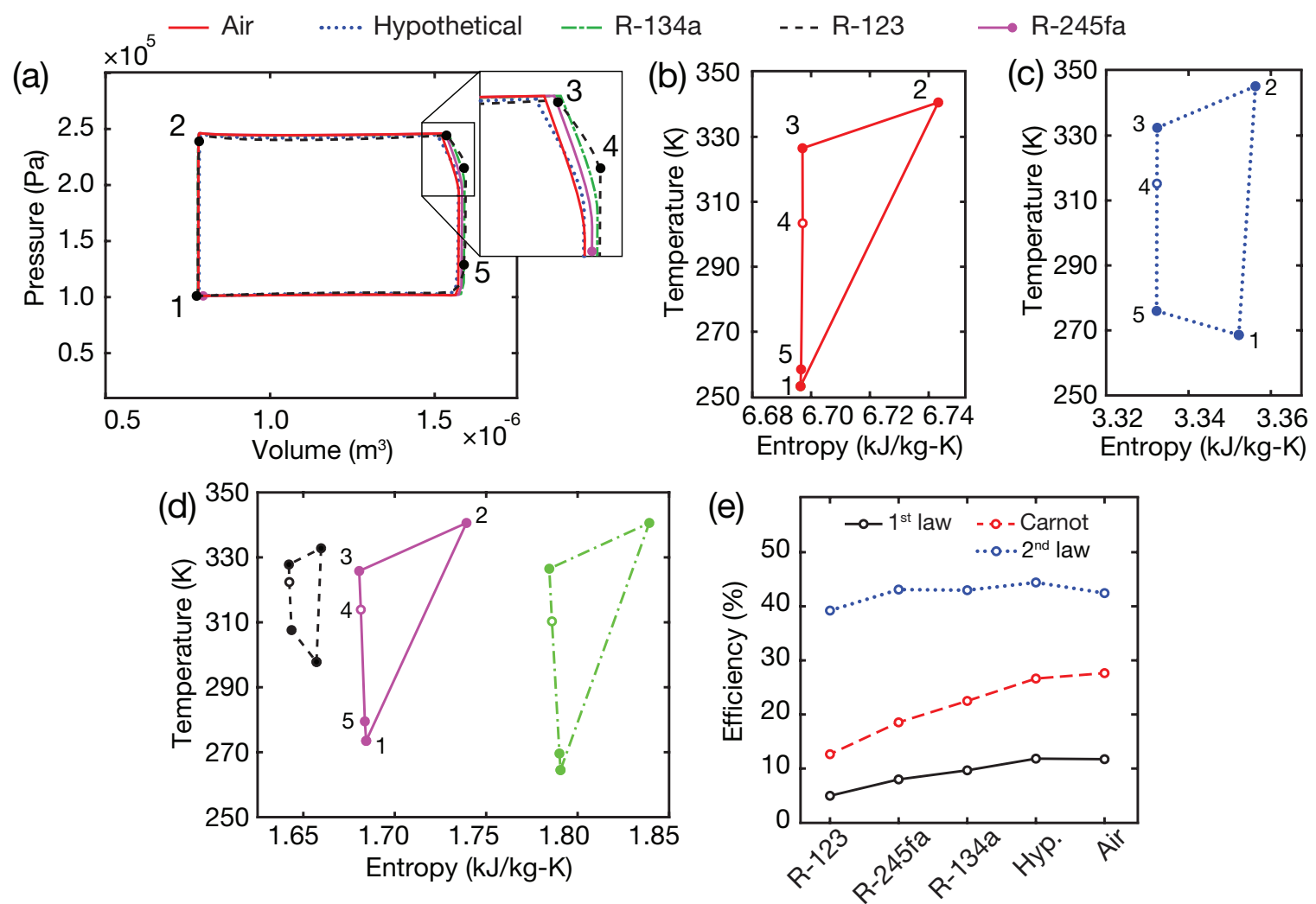

Figure 8: (a) Pressure-Volume diagrams (b,c,d) Temperature-Entropy diagrams (e) Plot of different efficiencies for different working fluids with $P_{i}=250 \mathrm{kPa}, T_{i}=323 \mathrm{~K}, \bar{t}_{13}=3$, and $\chi=0.02$. Here, the corresponding first law, second law, and Carnot efficiencies [21] are presented. Note that the temperature of the working fluid exiting the FPE are as low as 254 (air), 257 (hypothetical), 264 (R-134a), 273 (R-245fa), and 292 (R-123) K.

observations are made: (1) the working fluids (low $\mu$ ) used in the study - commonly employed working fluids - offer minimal resistance $b_{f}$ compared to the external load $b_{e m}$ on the FPE, (2) an increase in $\gamma$, increases the efficiency of the FPE proportionally, and (3) $c_{p}$ has no significant effect on the FPE efficiency. The study shows that to achieve a higher FPE efficiency, it is desirable for a working fluid to have high $\gamma$. In addition, the effect of leakage losses on the FPE showed that a leakage loss factor $\chi=0.02$ is found to be acceptable without any detrimental effects on FPE efficiency ( $<2 \%$ drop). The model predicts that by coupling a centimeter-scale electromagnetic energy converter, a peak output voltage of about $20 \mathrm{~V}$ and peak output power of about $2 \mathrm{~W}$ can be generated, subsequently offering the promise of reliable power generation at miniature length scales from low temperature waste heat sources.

\section{Appendix}

To demonstrate that a low $\beta$ results in the deviation of the constant pressure and constant volume processes, two P-V diagrams are generated for the reference case. One, generated for a constricted valve (low $\beta, \beta=0.00032 \mathrm{~kg} / \mathrm{Pa}-\mathrm{s}$ ) and other for the reference valve (high $\beta, \beta=0.0064 \mathrm{~kg} / \mathrm{Pa}$-s) with air as working fluid and operating conditions: $P_{i}=250 \mathrm{kPa}$, $T_{i}=323 \mathrm{~K}, \bar{t}_{13}=3$, and $\chi=0.02$. Note that the FPE energy conversion efficiencies for low and high $\beta$ are 


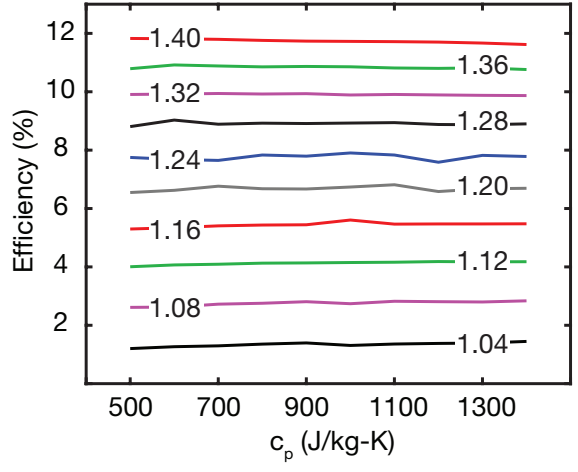

Figure 9: Effect of fluid properties $\gamma \in[1.04,1.40]$ and $c_{p} \in$ $[500,1400] \mathrm{J} / \mathrm{kg}-\mathrm{K}$ on the FPE for the reference case with $P_{i}=250 \mathrm{kPa}, T_{i}=323 \mathrm{~K}, \bar{t}_{13}=3$, and $\chi=0.02$.

$9.35 \%$ and $11.72 \%$, respectively. In addition a constricted valve (low $\beta$ ) promotes the retention of the expended working fluid in the cylinder for a longer duration, decreasing the operating frequency from $17.3 \mathrm{~Hz}$ (high $\beta$ ) to $5.1 \mathrm{~Hz}$ (low $\beta$ ).

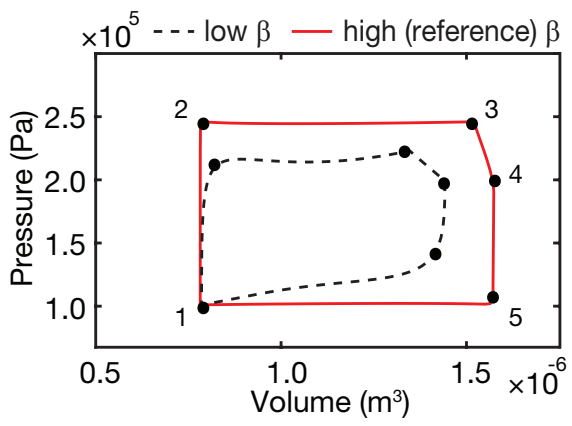

Figure A1: Comparison of two P-V diagrams for the reference case with low $\beta(0.00032 \mathrm{~kg} / \mathrm{Pa}-\mathrm{s})$ and high $\beta(0.0064 \mathrm{~kg} / \mathrm{Pa}-$ s) and air as working fluid with operating conditions: $P_{i}=250$ $\mathrm{kPa}, T_{i}=323 \mathrm{~K}, \bar{t}_{13}=3$, and $\chi=0.02$.

\section{Acknowledgment}

This work was supported in part by Wichita State University capitalization funds, and by the NSF via cooperative agreement OIA-1541079.

\section{Nomenclature}

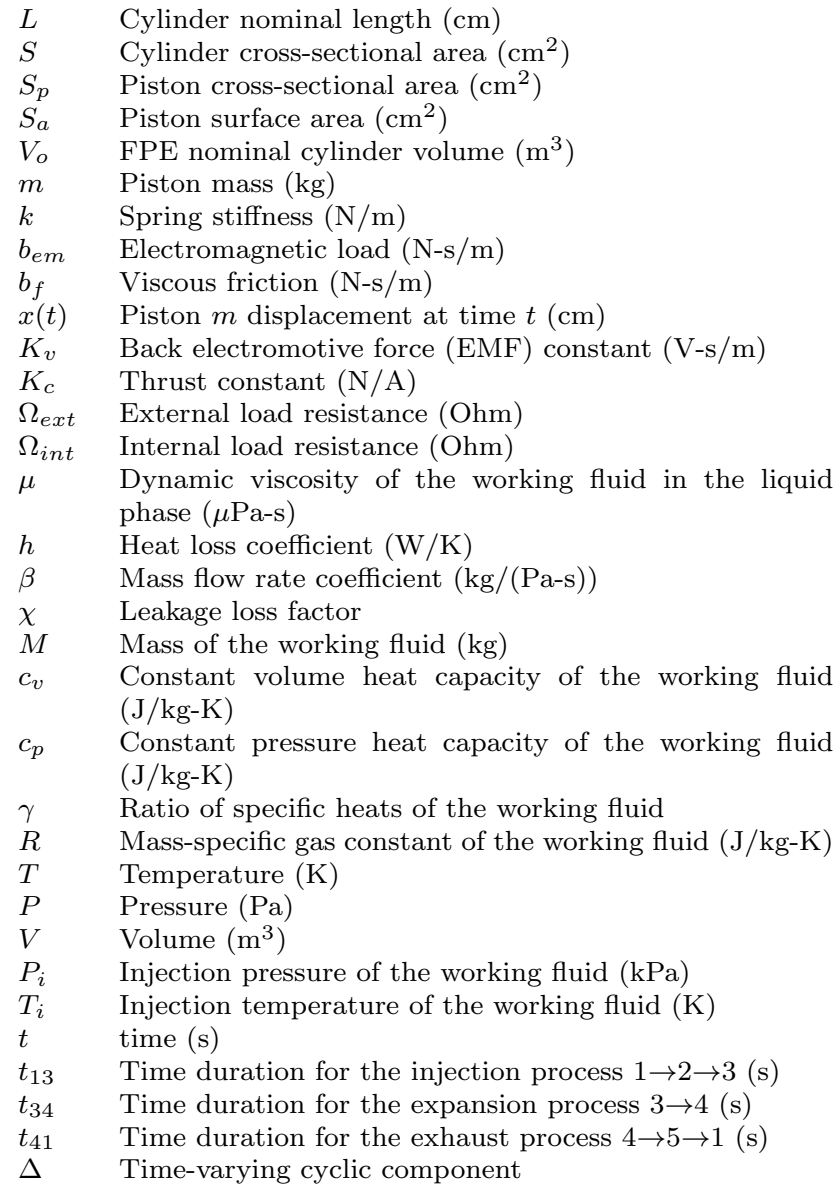

Abbreviations

FPE: Free piston expander EMF: Electromotive force

TDC: Top dead center

BDC: Bottom dead center

$\mathrm{CV}$ : Cylinder or control volume

Other Subscripts and Superscripts

Numerical subscript FPE's thermodynamic state

Overbar Nondimensional term

Overdot Differentiation with respect to time

Subscript o Ambient

Subscript $i$ Injection

Subscript e Exhaust

Subscript $l$ Leakage 


\section{References}

[1] S. P. Burugupally, L. Weiss, Power generation via small length scale thermo-mechanical systems: Current status and challenges, a review Energies 11 (9). doi:10.3390/ en11092253.

URL http://www.mdpi .com/1996-1073/11/9/2253

[2] D. C. Walther, J. Ahn, Advances and challenges in the development of power-generation systems at small scales, Progress in Energy and Combustion Science 37 (5) (2011) 583-610.

[3] B. Preetham, L. Weiss, Investigations of a new free piston expander engine cycle, Energy 106 (2016) 535 - 545. doi: $10.1016 / \mathrm{j}$. energy.2016.03.082

[4] H. Bardaweel, B. S. Preetham, R. Richards, C. Richards, M. Anderson, Mems-based resonant heat engine: scaling analysis Microsystem Technologies 17 (8) (2011) 12511261. doi:10.1007/s00542-011-1306-y URL https://doi.org/10.1007/s00542-011-1306-y

[5] L. Weiss, Power production from phase change in mems and micro devices, a review, International Journal of Thermal Sciences 50 (5) (2011) 639-647.

[6] J. Heywood, Internal Combustion Engine Fundamentals McGraw-Hill Education, 1988.

URL https://books.google.com/books?id= U9FSAAAAMAAJ

[7] E. Sher, I. Sher, Theoretical limits of scaling-down internal combustion engines, Chemical Engineering Science 66 (3) (2011) 260 - 267. doi:https://doi.org/10.1016/ j.ces.2010.10.005

URL http://www.sciencedirect.com/science/article/ pii/S0009250910005919

[8] Y. Tian, H. Zhang, G. Li, X. Hou, F. Yu, F. Yang, Y. Yang, Y. Liu, Experimental study on free piston linear generator (FPLG) used for waste heat recovery of vehicle engine, Applied Thermal Engineering 127 (Supplement C) (2017) 184 - 193. doi:10.1016/j.applthermaleng. 2017.08 .031

[9] B. S. Preetham, M. Anderson, C. Richards, Modeling of a resonant heat engine, Journal of Applied Physics 112 (12). doi:http://dx.doi.org/10.1063/1.4769447

[10] B. Preetham, M. Anderson, C. Richards, Estimation of parasitic losses in a proposed mesoscale resonant engine: Experiment and model, Journal of Applied Physics 115 (5) (2014) 054904.

[11] G. Li, H. Zhang, F. Yang, S. Song, Y. Chang, F. Yu, J. Wang, B. Yao, Preliminary development of a free piston expander-linear generator for small-scale organic rankine cycle (orc) waste heat recovery system, Energies 9 (4).
[12] S. P. Burugupally, L. Weiss, Design and performance of a miniature free piston expander, Energy 170 (2019) 611 618. doi:https://doi.org/10.1016/j.energy.2018.12. 158

URL http://www.sciencedirect.com/science/article/ pii/S0360544218325325

[13] C. Champagne, L. Weiss, Performance analysis of a miniature free piston expander for waste heat energy harvesting, Energy Conversion and Management 76 (2013) 883-892.

[14] S. Thapa, E. Borquist, A. Baniya, L. Weiss, Experimental and computational investigation of a mems-based boiler for waste heat recovery, Energy Conversion and Management 100 (2015) 403-413.

[15] X. Hou, H. Zhang, F. Yu, H. Liu, F. Yang, Y. Xu, Y. Tian, G. Li, Free piston expander-linear generator used for organic Rankine cycle waste heat recovery system, Applied Energy 208 (Supplement C) (2017) 1297 - 1307. doi:10.1016/j.apenergy.2017.09.024

[16] X. Hou, H. Zhang, Y. Xu, Y. Tian, T. Zhao, J. Li, F. Yu, Performance investigation of a free piston expander-linear generator for small scale organic rankine cycle, Applied Thermal Engineering 144 (2018) 209 - 218. doi:https: //doi.org/10.1016/j.applthermaleng.2018.08.059 URL http://www.sciencedirect.com/science/article/ pii/S135943111831370X

[17] C. Sprouse, C. Depcik, Review of organic rankine cycles for internal combustion engine exhaust waste heat recovery Applied Thermal Engineering 51 (1) (2013) 711 722. doi:https://doi.org/10.1016/j.applthermaleng. 2012.10.017.

URL http://www.sciencedirect.com/science/article/ pii/S1359431112006734

[18] A. Tavakolpour-Saleh, S. Zare, A. Omidvar, Applying perturbation technique to analysis of a free piston stirling engine possessing nonlinear springs, Applied Energy 183 (2016) 526 - 541. doi:https://doi.org/10.1016/j. apenergy.2016.09.009

URL http://www.sciencedirect.com/science/article/ pii/S0306261916313113

[19] J. Xiao, Q. Li, Z. Huang, Motion characteristic of a free piston linear engine Applied Energy 87 (4) (2010) $1288-$ 1294. doi:https://doi.org/10.1016/j.apenergy. 2009 . 07.005

URL http://www.sciencedirect.com/science/article/ pii/S0306261909002943

[20] B. Jia, Z. Zuo, G. Tian, H. Feng, A. Roskilly, Development and validation of a free-piston engine generator numerical model Energy Conversion and Management 91 (2015) 333 - 341. doi:https://doi.org/10.1016/j. enconman.2014.11.054 
URL http://www.sciencedirect.com/science/article/ pii/S0196890414010231

[21] Y. Çengel, M. Boles, Thermodynamics: An Engineering Approach, McGraw-Hill Education, 2014.

URL https://books.google.com/books?id= C1qFoAEACAAJ 